\title{
Possibly interacting Vorontsov-Vel'yaminov galaxies
}

\section{Observations of VV 432, VV 543 and VV 747}

\author{
A.V. Zasov ${ }^{1}$, A.Y. Kniazev ${ }^{2}$, S.A. Pustilnik ${ }^{2}$, A.G. Pramsky ${ }^{2}$, A.N. Burenkov ${ }^{2}$, A.V. Ugryumov ${ }^{2}$, and J.-M. Martin ${ }^{3}$ \\ 1 State Astronomical Institute of the Moscow State University, Universitetsky Pr. 13, Moscow 119899, Russia \\ e-mail: zasov@sai.msu.su \\ 2 Special Astrophysical Observatory, Nizhnij Arkhyz, Karachai-Circessia 357147, Russia \\ e-mail: akn@sao.ru sap@sao.ru pramsky@sao.ru ban@sao.ru and@sao.ru \\ 3 Département de Radioastronomie ARPEGES, Observatoire de Paris, F-92195 Meudon Cedex, France \\ e-mail: jmmartin@obspm.fr
}

Received December 15, 1999; accepted March 27, 2000

\begin{abstract}
Among the galaxies which were included in the Atlas and Catalogue of Interacting galaxies by VorontsovVel'yaminov (hereafter VV) as multiple systems ("nests", "chains" and similarly looking systems), there are many objects, where the interaction is not evident. Some of them are single objects, including low-mass galaxies with active star formation (SF). In this work we present the description of observations and results of the long-slit spectrophotometry with the Russian $6 \mathrm{~m}$ telescope of three VV-galaxies looking like double or multiple systems, and Hi observations of one of them in order to elucidate their nature, determine their metallicity, kinematic properties and the evolution status. Galaxies VV 432 and VV 747 are found to be dwarf systems with low oxygen abundance $(\mathrm{O} / \mathrm{H} \approx 1 / 22$ and $1 / 12$ of the solar value, respectively). Their velocity curves indicate quite slow rotation with respective maximum velocities of about 60 and $80 \mathrm{~km} \mathrm{~s}^{-1}$, in agreement with their low luminosities. The distance to VV 432 is rather uncertain. If it is a member of Virgo Cluster, this is the most metal-deficient known galaxy of this aggregate. For galaxy VV 543 the measured emissionline redshift 0.047 appeared ten times larger than it was given in the original paper and is cited in databases. This "system" evidently represents an optical pair of two galaxies with large velocity difference. The fainter western component is an Hil-galaxy, while the brighter one is an absorption-line early-type galaxy with the radial velocity being $1600 \mathrm{~km} \mathrm{~s}^{-1}$ lower.
\end{abstract}

Key words: galaxies: interacting — galaxies: abundances — galaxies: starburst — galaxies: individual (VV 432, VV 543, VV 747)

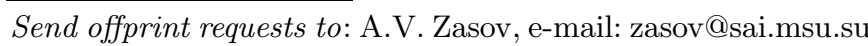

\section{Introduction}

Among the definitely interacting galaxies in close pairs or groups, many of which were discovered by VorontsovVel'yaminov and included in his Atlas and Catalog of interacting galaxies (Vorontsov-Vel'yaminov 1959, 1977) there are objects having VV numbers, in which the interaction is not so evident. Such objects were usually classified by Vorontsov-Vel'yaminov as the "nests" or often similarly looking systems called "chains" or "pairs in contact". He considered these objects as compact fragmenting systems, giving birth to young galaxies, but very soon observations showed that their nature may be different. Such systems can present both galaxies of strange and unusual shapes (either single objects or mergers), and really multiple systems, where it is hard to guess the number of components without detailed studies. In many cases only spectral measurements of their gas velocity distribution enable to prove or to disprove their solitude.

Earlier spectral observations confirmed that some VV systems actually present single dwarf galaxies with clumpy inner structure, resembling dwarf irregulars with multiple regions of active star formation (SF), or blue compact galaxies (BCGs) (Vorontsov-Vel'yaminov 1979a,b; Afanasiev et al. 1980; Arkhipova et al. 1981, 1987a-c). Only a few single nearby VV galaxies have been studied in detail (e.g. VV $556 \equiv$ GR 8, VV $499 \equiv$ DDO 053).

The important questions on the chemical abundances in the studied VV galaxies left outside the scope of these early observations. New opportunities appeared due to new CCD-detectors, which enable to realize high sensitivity and large dynamical range. It induced authors to return back to the study of "nest-like" VV galaxies. Besides, many new observational data have appeared on many of these objects. 
For the current study we selected more than twenty VV galaxies looking like multiple systems or singular irregular systems in the POSS/DSS ${ }^{1}$ images, for which such indicators of interaction as well defined tidal tails or bridges are absent. The main objectives of our investigation are:

- to clarify the distances, total luminosities and masses of those systems, systemic velocities of which are not known or badly known;

- to carry out high $S / N$ ratio spectrophotometry in order to address problems of chemical abundances and evolutional status of these objects;

- to analyse the inner gas kinematics and structural properties of the objects.

Since many of VV galaxies in question are dwarfs with recent or current SF burst, it is also important to check possible companions which could exert strong enough tidal action.

In this paper, the first in the series, we present the results of recent long-slit spectroscopy for three VV objects: VV 432, VV 543 and VV 747 and the observation in HI-line of VV 747.

In Sect. 2 we describe observations, data reduction, abundances determination and the measurements of velocity distribution of ionized gas along the slit.

Observations of VV 747 in the HI-line $21 \mathrm{~cm}$ and their results are presented in Sect. 3. In Sect. 4 we consider the individual properties of studied galaxies. Discussions and preliminary conclusions are presented in Sect. 5. We adopt throughout the paper $H_{0}=75 \mathrm{~km} \mathrm{~s}^{-1}$.

\section{Spectral observations, data reduction and analysis}

\subsection{Observations}

The spectroscopic data were obtained with the $6 \mathrm{~m}$ telescope of the Special Astrophysical Observatory of Russian Academy of Science (SAO RAS) during two runs in February and April 1999. The Long-Slit spectrograph (LSS in Table 1) (Afanasiev et al. 1995) at the telescope prime focus was equipped with a Photometrics CCDdetector PM1024 (with $24 \times 24 \mu \mathrm{m}$ pixel size) (PMCCD in Table 1) installed at Schmidt-Cassegrain camera F/1.5. Most of the long-slit spectra $\left(1.2^{\prime \prime} \times 180^{\prime \prime}\right)$ were obtained with the grating of 325 grooves $/ \mathrm{mm}$, giving a dispersion of $4.6 \AA /$ pixel. Additional data were obtained with the grating of 1302 grooves/mm and dispersion $1.2 \AA /$ pixel. For the latter set-up the slit of $2^{\prime \prime} \times 180^{\prime \prime}$ was used. The scale along the slit was $0.39^{\prime \prime} /$ pixel.

The resulting resolution (FWHM) was about $14-15 \AA$ for the first set-up, and about $3.7 \AA$ for the second setup. Reference spectra of an $\mathrm{Ar}-\mathrm{Ne}-\mathrm{He}$ lamp were recorded

\footnotetext{
1 DSS is Digital Sky Survey distributed by Hubble Space Telescope Science Institute.
}

before or after each observation to provide wavelength calibration. Spectrophotometric standard stars from Massey et al. (1988) were observed for flux calibration at least twice a night.

Observations and data processing in this set-up have been conducted mainly under the software package NICE in MIDAS, described by Kniazev \& Shergin (1995).

\subsection{Data reduction and abundances determination}

The data reduction was performed in SAO RAS, using various packages of MIDAS (see Kniazev et al. 2000, for details).

In Table 2 we summarize the main observational parameters of the discussed three VV "nests". They include the names of the objects, their coordinates for the epoch J2000, the apparent blue magnitudes and the corresponding references, the radial heliocentric velocities, measured in this work with their rms uncertainties, maximal angular sizes, absolute blue magnitudes and the oxygen abundances $(12+\log (\mathrm{O} / \mathrm{H}))$.

Direct images of studied galaxies, extracted from the DSS and the position of long slit, indicated by bar are presented in Figs. 1a, 2a, 3a. Corresponding 2-D spectra are shown in Figs. 1c, 2c, 3c. The brightness profiles of $\mathrm{H} \alpha$ line along the slit, and corresponding velocity curves are illustrated in Figs. 1b, 2b, 3b. In Figs. 1d, 2d, and $3 \mathrm{~d}$ we present 1-D spectra, extracted from 2-D spectra, which were used for the measurements of line intensities, determination of physical conditions and abundances of HiI-regions.

The resulting observed emission line intensities $F(\lambda)$ of various ions relative to $\mathrm{H} \beta$, both uncorrected and corrected for interstellar extinction and underlying stellar absorption $I(\lambda)$ (following the procedure described by Izotov et al. 1997) for the brightest parts of the galaxies are presented in Tables 3 along with the extinction coefficient $C(\mathrm{H} \beta)$, the equivalent width of absorption Balmer hydrogen lines $\mathrm{EW}(\mathrm{abs})$, the equivalent width of $\mathrm{H} \beta$ line $\mathrm{EW}(\mathrm{H} \beta)$ and the observed $\mathrm{H} \beta$ flux.

For the abundances determination we used the scheme, described in detail by Izotov et al. (1994, 1997). The electron temperatures and densities in HiI-regions of the observed VV-galaxies and their abundances of $\mathrm{O}, \mathrm{N}$ and $\mathrm{Ne}$ are summarized in Table 4.

For VV 543 the [OIII]-line $4363 \AA$ is not detected. Therefore to estimate its metallicity we employ the empirical method (see e.g. Pagel et al. 1979; McGaugh 1991; Olofsson 1997). Its uncertainty for $12+\log (\mathrm{O} / \mathrm{H})$ can be as large as $0.2-0.3$ dex. We also applied this empirical method to all four individual knots of VV 432.

The ratio $[\mathrm{OIII}] /[\mathrm{NII}]$ of extinction corrected intensities of the lines $\lambda 4959,5007 \AA$ and $\lambda 6548,6584 \AA$ enable one to avoid well known ambiguity of the empirical method (Alloin et al. 1979). Taking into account these ratios, one 
Table 1. Journal of observations

\begin{tabular}{ccccrcrr}
\hline $\begin{array}{c}\text { Galaxy } \\
\text { Name } \\
(1)\end{array}$ & Date & Instrument & $\begin{array}{c}\text { Grating } \\
\text { grooves } / \mathrm{mm}] \\
(4)\end{array}$ & $\begin{array}{c}\text { Exposure } \\
\text { time }[\mathrm{s}] \\
(5)\end{array}$ & $\begin{array}{c}\text { Wavelength } \\
\text { Range }[\AA] \\
(6)\end{array}$ & $\begin{array}{c}\text { Dispersion } \\
{[\AA / \text { pixel] }} \\
(7)\end{array}$ & $\begin{array}{c}\text { PA } \\
{[\text { Degree }]}\end{array}$ \\
\hline VV 432 & 12.02 .1999 & LSS+PMCCD & 325 & 900 & $3700-8000$ & 4.6 & 27 \\
VV 432 & 23.04 .1999 & LSS+PMCCD & 1302 & $2 \times 1200$ & $4000-5200$ & 1.2 & 27 \\
VV 432 & 23.04 .1999 & LSS+PMCCD & 1302 & $2 \times 1200$ & $6000-7200$ & 1.2 & 27 \\
VV 543 & 11.02 .1999 & LSS+PMCCD & 325 & 900 & $3700-8000$ & 4.6 & 101 \\
VV 747 & 12.02 .1999 & LSS+PMCCD & 325 & 1200 & $3700-8000$ & 4.6 & 56 \\
\hline
\end{tabular}

Table 2. Main parameters of studied VV-galaxies

\begin{tabular}{|c|c|c|c|c|}
\hline Parameter & VV 432 & VV $543 \mathrm{~W}$ & VV $543 \mathrm{E}$ & VV 747 \\
\hline$\overline{\alpha_{2000}}$ & $12^{\mathrm{h}} 17^{\mathrm{m}} 34.7^{\mathrm{s}}$ & $13^{\mathrm{h}} 42^{\mathrm{m}} 22.2^{\mathrm{s}}$ & $13^{\mathrm{h}} 42^{\mathrm{m}} 23.5^{\mathrm{s}}$ & $10^{\mathrm{h}} 57^{\mathrm{m}} 46.9^{\mathrm{s}}$ \\
\hline$\delta_{2000}$ & $+12^{\circ} 23^{\prime} 46^{\prime \prime}$ & $+29^{\circ} 49^{\prime} 33^{\prime \prime}$ & $+29^{\circ} 49^{\prime} 30^{\prime \prime}$ & $+36^{\circ} 15^{\prime} 38^{\prime \prime}$ \\
\hline$B_{\text {tot }}^{\mathrm{L}}$ & $14.73 \pm 0.15$ & $17.7 \pm 0.3$ & $15.20 \pm 0.20$ & $15.52 \pm 0.71$ \\
\hline$A_{\mathrm{B}}^{\mathrm{N}}$ & 0.03 & 0.00 & 0.00 & 0.04 \\
\hline$V_{\mathrm{HeI}}\left(\mathrm{km} \mathrm{s}^{-1}\right)$ & $-160 \pm 6$ & $14100 \pm 20$ & $12480 \pm 90$ & $629 \pm 7^{5}$ \\
\hline $\operatorname{Dist}_{\text {Vir }}(\mathrm{Mpc})$ & $20.7 \pm 0.8$ & $188.4 \pm 0.3$ & $166.8 \pm 1.2$ & $9.2 \pm 0.1$ \\
\hline$M_{B}^{1}$ & -16.8 & -18.6 & -20.9 & -14.4 \\
\hline$D_{25}(\operatorname{arcsec})$ & 111 & $\approx 16$ & 39 & 50 \\
\hline$D_{25}(\mathrm{kpc})$ & 11.1 & $\approx 14.6$ & 31.5 & 2.2 \\
\hline Axis ratio $b / a^{L}$ & 0.31 & 0.50 & 1.00 & 0.85 \\
\hline $12+\log (\mathrm{O} / \mathrm{H})$ & $7.58 \pm 0.06$ & $8.5 \pm 0.1$ & - & $7.85 \pm 0.05$ \\
\hline Hi flux ${ }^{2}$ & $9.04 \pm 1.99^{3}$ & - & - & $4.03 \pm 0.39$ \\
\hline$W_{20} \mathrm{~km} \mathrm{~s}^{-1}$ & $123^{3}$ & - & - & $117 \pm 11$ \\
\hline$M(\mathrm{HI})\left(10^{8} M_{\odot}\right)$ & $9.1 \pm 2.0$ & - & - & $0.81 \pm 0.08$ \\
\hline$M(\mathrm{HI}) / L_{B}^{4}$ & 1.2 & - & - & 1.0 \\
\hline $\begin{array}{l}B_{\text {tot }}-\text { total blue } \\
D_{25}-\text { Diameter } \\
A_{B}-\text { Galactic e } \\
1 \text { With Galactic } \\
3 \text { From Schneide }\end{array}$ & $\begin{array}{l}\text { magnitude; } M_{I} \\
\text { t surface brigh } \\
\text { tinction; }{ }^{L} \text { Da } \\
\text { xtinction correc } \\
\text { et al. (1991); }{ }^{4}\end{array}$ & $\begin{array}{l}- \text { absolute } \mathrm{b} \\
\text { ness } \mu_{B}=25 \\
\text { a from LEDA; } \\
\text { tion; }{ }^{2} \text { Units } \\
\text { In }\left(M / L_{B}\right) \odot ;\end{array}$ & $\begin{array}{l}\text { gnitude. } \\
\text { t". } \\
\text { ta from NED. } \\
\left.\mathrm{s}^{-1}\right) \text {. }\end{array}$ & \\
\hline
\end{tabular}

Table 3. Line intensities in the knot "a" of VV 432, VV 747 and VV 543W

\begin{tabular}{|c|c|c|c|c|c|c|c|c|}
\hline \multirow[b]{2}{*}{$\lambda_{0}(\AA)$} & \multicolumn{2}{|c|}{ VV 432 (a) $(4.6 \AA /$ pixel $)$} & \multicolumn{2}{|c|}{ VV 432 (a) $(1.2 \AA /$ pixel $)$} & \multicolumn{2}{|c|}{ VV 747 (a) $(4.6 \AA ̊$ pixel $)$} & \multicolumn{2}{|c|}{ VV 543W $(4.6 \AA /$ pixel $)$} \\
\hline & $F(\lambda) / F(\mathrm{H} \beta)$ & $I(\lambda) / I(\mathrm{H} \beta)$ & $F(\lambda) / F(\mathrm{H} \beta)$ & $I(\lambda) / I(\mathrm{H} \beta)$ & $F(\lambda) / F(\mathrm{H} \beta)$ & $I(\lambda) / I(\mathrm{H} \beta)$ & $F(\lambda) / F(\mathrm{H} \beta)$ & $I(\lambda) / I(\mathrm{H} \beta)$ \\
\hline $3727[\mathrm{O}$ II $]$ & $2.17 \pm 0.10$ & $2.63 \pm 0.12$ & ( $1=1$ & $1,1-1$ & $1.08 \pm 0.06$ & $1.57 \pm 0.10$ & $3.79 \pm 0.33$ & $4.31 \pm 0.40$ \\
\hline 3868 [Ne III] & $0.33 \pm 0.03$ & $0.39 \pm 0.04$ & - & - & $0.30 \pm 0.01$ & $0.40 \pm 0.02$ & - & - \\
\hline $4101 \mathrm{H} \delta$ & $0.26 \pm 0.02$ & $0.30 \pm 0.04$ & $0.23 \pm 0.03$ & $0.26 \pm 0.04$ & $0.22 \pm 0.01$ & $0.28 \pm 0.02$ & - & \\
\hline $4340 \mathrm{H} \gamma$ & $0.49 \pm 0.02$ & $0.54 \pm 0.03$ & $0 \pm 0.03$ & $0.54 \pm 0.04$ & $0.40 \pm 0.01$ & $0.47 \pm 0.02$ & $0.50 \pm 0.07$ & $0.53 \pm 0.10$ \\
\hline 4363 [O & $0.11 \pm 0.01$ & $0.12 \pm 0.02$ & $0.09 \pm 0.03$ & $0.09 \pm 0.03$ & $0.09 \pm 0.01$ & $0.11 \pm 0.01$ & & \\
\hline $4861 \mathrm{H} \beta$ & $1.00 \pm 0.02$ & $1.00 \pm 0.03$ & $1.00 \pm 0.04$ & $1.00 \pm 0.04$ & $1.00 \pm 0.01$ & $1.00 \pm 0.02$ & $1.00 \pm 0.09$ & $1.00 \pm 0.11$ \\
\hline 4959 [O III] & $1.10 \pm 0.02$ & $1.08 \pm 0.02$ & $1.09 \pm 0.04$ & $1.07 \pm 0.04$ & $1.79 \pm 0.18$ & $1.74 \pm 0.18$ & $0.46 \pm 0.08$ & $0.45 \pm 0.08$ \\
\hline 5007 [O III] & $3.36 \pm 0.05$ & $3.28 \pm 0.05$ & $3.25 \pm 0.10$ & $3.18 \pm 0.09$ & $5.49 \pm 0.23$ & $5.27 \pm 0.22$ & $1.26 \pm 0.11$ & $1.23 \pm 0.11$ \\
\hline $5876 \mathrm{He} \mathrm{I}$ & $0.09 \pm 0.02$ & $0.08 \pm 0.02$ & - & - & $0.09 \pm 0.01$ & $0.07 \pm 0.01$ & - & \\
\hline 6548 [N II] & $0.05 \pm 0.01$ & $0.05 \pm 0.01$ & $0.03 \pm 0.01$ & $0.03 \pm 0.01$ & $0.03 \pm 0.01$ & $0.02 \pm 0.00$ & $0.20 \pm 0.03$ & $0.17 \pm 0.02$ \\
\hline $6563 \mathrm{H} \alpha$ & $3.39 \pm 0.07^{1}$ & $2.74 \pm 0.06$ & $3.39 \pm 0.10$ & $2.77 \pm 0$ & $2.34 \pm 0.04$ & $2.79 \pm 0.05^{2}$ & $3.35 \pm 0.23$ & $2.88 \pm 0.22$ \\
\hline 6584 [N II] & $0.14 \pm 0.04$ & $0.11 \pm 0.03$ & $0.11 \pm 0.01$ & $0.09 \pm 0.01$ & $0.09 \pm 0.03$ & $0.06 \pm 0.02$ & $0.74 \pm 0.08$ & $0.64 \pm 0.07$ \\
\hline $6717[\mathrm{~S}$ II] & $0.26 \pm 0.02$ & $0.21 \pm 0.01$ & $0.29 \pm 0.02$ & $0.23 \pm 0.01$ & $0.13 \pm 0.01$ & $0.09 \pm 0.01$ & $0.99 \pm 0.11$ & $0.84 \pm 0.10$ \\
\hline 6731 [S II] & $0.17 \pm 0.01$ & $0.13 \pm 0.01$ & $0.20 \pm 0.02$ & $0.16 \pm 0.01$ & $0.10 \pm 0.01$ & $0.07 \pm 0.01$ & $0.60 \pm 0.10$ & $0.51 \pm 0.09$ \\
\hline$C(\mathrm{H} \beta) \operatorname{dex}$ & \multicolumn{2}{|c|}{$0.275 \pm 0.026$} & \multicolumn{2}{|c|}{$0.265 \pm 0.037$} & \multicolumn{2}{|c|}{$0.445 \pm 0.016$} & \multicolumn{2}{|c|}{$0.190 \pm 0.088$} \\
\hline EW(abs) $\AA$ & \multirow{2}{*}{\multicolumn{2}{|c|}{$\begin{array}{l}0.100 \pm 1.807 \\
123.10 \pm 1.81\end{array}$}} & \multirow{2}{*}{\multicolumn{2}{|c|}{$\begin{array}{c}0.050 \pm 2.067 \\
91.08 \pm 2.54\end{array}$}} & \multirow{2}{*}{\multicolumn{2}{|c|}{$0.080 \pm 0.985$}} & \multirow{2}{*}{\multicolumn{2}{|c|}{$0.100 \pm 0.827$}} \\
\hline$F(\mathrm{H} \beta)$ & & & & & & & $7.78 \pm$ & \\
\hline $\mathrm{EW}(\mathrm{H} \beta) \AA$ & \multicolumn{2}{|c|}{$120.96 \pm 1.78$} & \multicolumn{2}{|c|}{$128.37 \pm 3.58$} & \multicolumn{2}{|c|}{$125.46 \pm 1.14$} & \multicolumn{2}{|c|}{$13.52 \pm 0.87$} \\
\hline
\end{tabular}

${ }^{1}$ Due to calibration problems the intensity of $\mathrm{H} \alpha$ is likely a bit overestimated. To check its effect we redone calculation of $\mathrm{O} / \mathrm{H}$ for $C(\mathrm{H} \beta)=0$. It increases $12+\log (\mathrm{O} / \mathrm{H})$ by $0.01 .^{2}$ - the intensity of $\mathrm{H} \alpha$ is recalculated from the recombination ratio to $\mathrm{H} \beta$. 
can use $R_{23}$ and $Q$ parameters to get more reliable estimate of O/H through the model curves by Olofsson (1997). By this way it was obtained for VV 543W the abundance $12+\log (\mathrm{O} / \mathrm{H})=8.5 \pm 0.1$, which is about 0.3 lower than the value, derived from McGaugh (1991) tracks, and seems to be in a better agreement with the abundance, expected for underluminous HiI-galaxies.

\subsection{Velocity curves}

To obtain line-of-sight velocity distribution along the slit we use the MIDAS programs, kindly presented to authors by D. Makarov.

To increase the accuracy of derived rotation curves the programs were modified to include additional corrections using close lines in the reference spectrum.

The procedure includes the following steps: 1) a linearisation of 2-D spectrum of the object and of the reference spectrum; 2) a measurement of the position of the $\mathrm{H} \alpha$ emission for each of the 250 position rows using Gaussian fitting; 3) an estimation of the background and $S / N$ ratio for the $\mathrm{H} \alpha$ emission in each row; 4) a similar measurement of the nearby line NeI $\lambda 6598.95 \AA$ in the reference spectrum and compiling the table of differences between the laboratory wavelength of this line and the measured one for each row; 5) a polynomial fitting of these differences and a determination of the residual scattering (rms);6) the application of this fitting polynomial to correct the measured wavelengths of $\mathrm{H} \alpha$. The resulting error of wavelengths measurements is determined by quadratic summing of the fitting rms, found in the previous step, and the measurement error of velocity for each point along the slit, estimated from Gaussian fitting. The latter varies between $2.5 \mathrm{~km} \mathrm{~s}^{-1}$ for the points with the highest signal and $9 \mathrm{~km} \mathrm{~s}^{-1}$ for the points with low $S / N$ ratio for dispersion $1.2 \AA /$ pixel; 7) rebinning of $\mathrm{H} \alpha$ data along the slit, corresponding to the seeing during the observations (4 or 5 pixels in April and February, respectively).

Below we use only those velocity estimates which satisfy the criteria $S / N>3$ and $\sigma_{V}<15 \mathrm{~km} \mathrm{~s}^{-1}$. Resulting high accuracy of corrected observed wavelength enables one to study irregularities of the velocity curve with an amplitude as low as $10 \mathrm{~km} \mathrm{~s}^{-1}$ on the angular scale up to $20^{\prime \prime}$.

For VV 432, the rotation curves which were obtained with the low and high resolution spectra are presented in Fig. 1b. Their similarity shows that our low dispersion spectra can be used to derive preliminary dynamical parameters of the studied galaxies.

\section{Hi data for VV 747}

For VV 747, the interpretation of the optical data remains ambiguous regarding its multiplicity (see Sect. 4.3).
In order to settle this ambiguity we investigated the dynamical state of the system and performed single-dish $\mathrm{HI}$ observations.

\subsection{Observations and data reduction}

The $21 \mathrm{~cm}$ HI-line observations were carried out in July 1999 with the Nançay ${ }^{2} 300 \mathrm{~m}$ telescope (NRT). The NRT has a half-power beam width of $3.7^{\prime}(\mathrm{EW}) \times 22^{\prime}(\mathrm{NS})$ at the declination $\delta=0^{\circ}$.

A dual polarization receiver has been used, with a system temperature of $\approx 40 \mathrm{~K}$ in the horizontal and vertical linear polarizations. The gain of the telescope was $1.1 \mathrm{~K}$ $\mathrm{Jy}^{-1}$ at a declination of $\delta=0^{\circ}$. The observations were made in position switching mode with 1-minute on-source and 1-minute off-source integrations.

Since VV 747 had a known optical redshift, we split the autocorrelator into two, each bank covering a bandwidth of $6.4 \mathrm{MHz}$, and centred at the frequency corresponding to the optical redshift. The velocity coverage was $1350 \mathrm{~km} \mathrm{~s}^{-1}$. The channel width was $2.6 \mathrm{~km} \mathrm{~s}^{-1}$ and after averaging pairs of adjacent channels the effective resolution was $6.3 \mathrm{~km} \mathrm{~s}^{-1}$.

The data has been reduced using the software developed by the telescope's staff. Both polarization spectra were calibrated and processed independently, and were averaged to improve the signal-to-noise ratio. Errors were calculated following Schneider et al. (1986).

\subsection{Results. HI-profile and its parameters}

For the total integration time of 234 minutes $(\mathrm{ON}+\mathrm{OFF}$ source) the rms noise is $4 \mathrm{mJy}$. The galaxy is detected with $S / N$ ratio of 12 . The spectrum is presented in Fig. 4 . The line shape is close to Gaussian and typical of lowmass galaxies. Its width at $50 \%$ of peak $W_{0.5}=90 \mathrm{~km} \mathrm{~s}^{-1}$ is close to the median value of the distribution derived for the statistical sample of BCGs from the zone of the Second Byurakan Survey (SBS) by Thuan et al. (1999). The line width at $20 \%$ of peak $W_{0.2}=117 \mathrm{~km} \mathrm{~s}^{-1}$ is very close to that of VV 432. This is important in the following discussion about the applicability of the Tully-Fisher relation (1977, hereafter TF) for the distance problem of the latter galaxy.

The HI-mass of VV 747 of $0.810^{8} M_{\odot}$, is typical of low luminosity gas-rich galaxies, like the ratio of the hydrogen mass over the blue luminosity: $M(\mathrm{HI}) / L_{B}=1.0$.

${ }^{2}$ The Nançay Radioastronomy Station is part of the Paris Observatory and is operated by the Ministère de l'Éducation Nationale and Institut des Sciences de l'Univers of the Centre National de la Recherche Scientifique. 

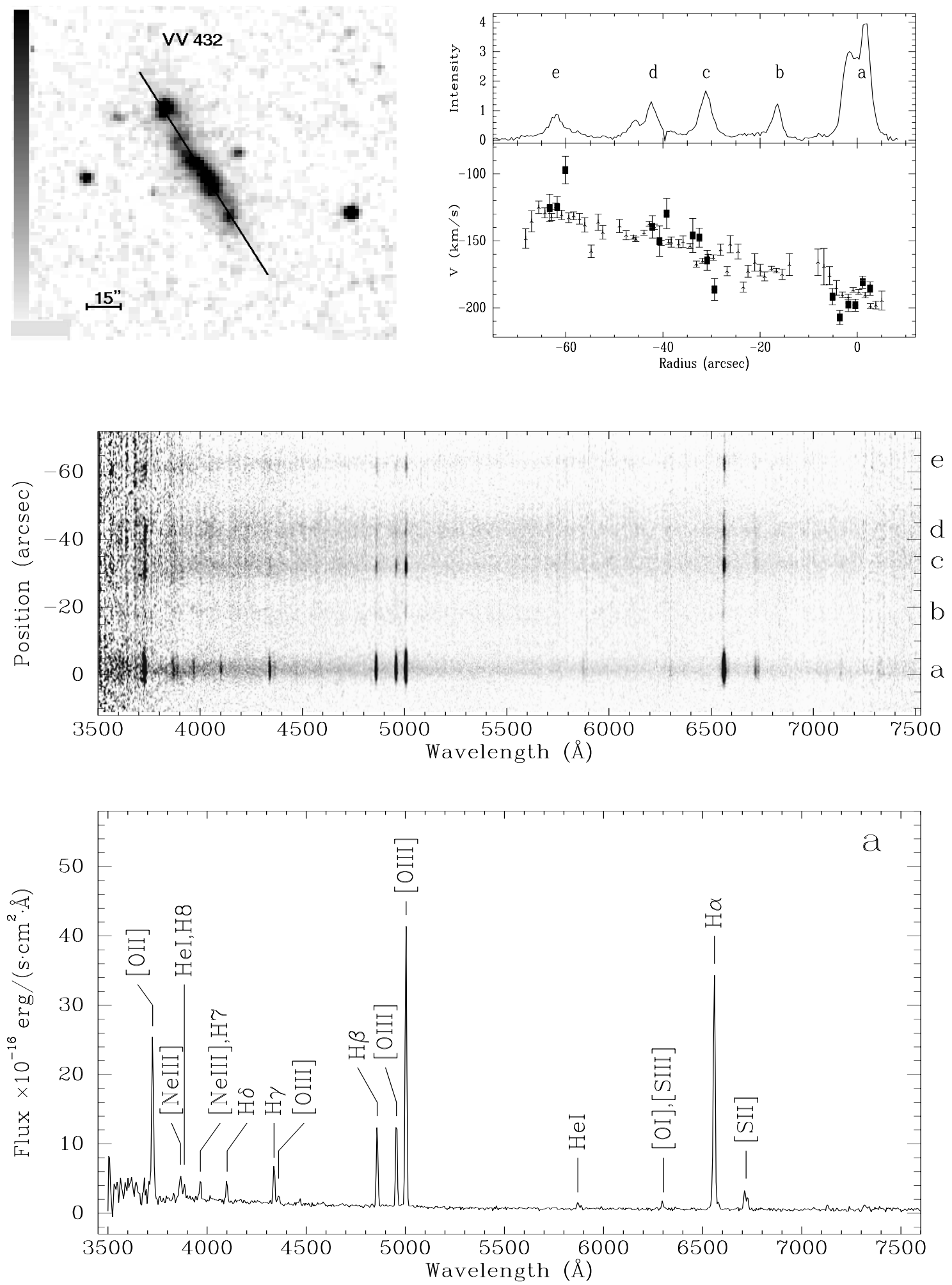

Fig. 1. From top-left to bottom: a) DSS image of galaxy VV 432 with the position of long slit superimposed. The spot "a" is NE bright region at the edge of the galaxy; b) Brightness profile of $\mathrm{H} \alpha$ in relative intensities along the slit and the velocity curve. Only independent along the slit points (accounting for seeing) from the spectra with dispersion $1.2 \AA /$ pixel and $4.6 \AA /$ pixel are shown by arrows and filled boxes respectively. The error bars correspond to $\pm 1 \sigma$. c) 2-D spectrum of VV 432 with dispersion $4.6 \AA$ /pixel; d) 1-D spectrum of the brightest NE spot ("a") 


\section{Properties of individual galaxies}

\section{1. $V V 432=I C 3105=U G C 7326$}

The first long-slit spectrum of this chain-like galaxy has been obtained along the major axis by Arkhipova et al. (1981). They revealed high-excitation spectrum of HiIregion in the NE region. The amplitude of the rotation curve has been found to be relatively small (less than about $60 \mathrm{~km} \mathrm{~s}^{-1}$ ).

Our long-slit spectrophotometry allowed to detect the NE HiI-region (knot "a" in Fig. 1) [OIII]-line $\lambda 4363 \AA$ with a good $S / N$ ratio in both spectra. The first spectrum, obtained on February 12, 1999 covered the range $3600-8000 \AA$ and the spectra taken on April 24, 1999 with a dispersion $1.2 \AA /$ pixel, covered two separate ranges $4000-5200 \AA$ and $6000-7200 \AA$. Table 3 presents the data for all measured emission lines. According to this table, the relative intensities of strong oxygen and hydrogen lines in both spectra are the same within $3 \%$, in accordance with their internal errors. For the abundance derivation we supposed that, for the April spectra, the relative intensity of the [OII]-line $\lambda 3727 \AA$ and of the $\mathrm{H} \beta$ line to be equal to those derived from the February spectrum.

Oxygen abundance determination gives consistent values for both data sets, so we accept the weighted mean of the two independent measurements. The resulting value of $12+\log (\mathrm{O} / \mathrm{H})=7.58 \pm 0.06$ obtained for this HII-region appear to be very low.

Since the [OIII]-line $\lambda 4363 \AA$ was not detected in other emission-line knots, the oxygen abundances for knots "b", "c" and "d" were estimated by the same empirical method mentioned in Sect. 2.3. The most uncertain parameter the mean gas density - was assumed to be close to that found for knot "a", what is about 1 atom $\mathrm{cm}^{-3}$ (the value, derived from the consistency of oxygen abundances, determined by classical and empirical methods). Oxygen abundances, found by the empirical method for this value of $N_{\mathrm{e}}$ for three knots, are consistent with the estimates, obtained for knot "a", taking into account the uncertainties of parameter $R_{23}$ and possible variations of the mean density $N_{\mathrm{e}}$ within the factor of 2 .

This galaxy is elongated (the axial ratio of $b / a \approx 1 / 3$ according to LEDA $)^{3}$, which is not untypical for dwarf galaxies. It looks like an edge-on disk, bent on both NE and SW edges, which probably indicates a tidal action from some other galaxy. The bright HiI-region on the NE edge appears to be outside of the main body of the galaxy, and its connection to the disk is not evident, although a smooth velocity distribution along the galaxy favours its interpretation as a single object. Another possibility which cannot be excluded is that the outer knots are large SF regions at the ends of spiral arms, seen not exactly edge-

\footnotetext{
${ }^{3}$ LEDA is the acronym of Lyon-Meudon Extragalactic Database, http://leda.univ-lyon1.fr
}

on. However due to the rather small luminosity of VV 432, the presence of spiral arms is quite improbable.

Our long-slit spectrum with the dispersion of $1.2 \AA /$ pixel allows to derive the rotation curve along the "disk", using the $\mathrm{H} \alpha$ line data (see Fig. 1b). A velocity gradient is well seen over the whole disk; there is no sign of flattening of the velocity curve on both NE and SW edges. The mean heliocentric velocity of the galaxy is $-160 \mathrm{~km} \mathrm{~s}^{-1}$. The amplitude of the rotation curve within the whole extent of $\mathrm{H} \alpha$-emission of about $70^{\prime \prime}$ corresponds to the maximal rotational velocity of $40 \pm 5 \mathrm{~km} \mathrm{~s}^{-1}$. The expected inclination correction is very small, since the disk is seen at the inclination angle $i>70^{\circ}$.

The uncertainties of the data points of the Fig. 1b's velocity curve are small and remain in the range 4 to $15 \mathrm{~km} \mathrm{~s}^{-1}$. The irregularities which are seen on the rotation curve can then be considered as real ones, probably connected with the regions of active SF.

The galaxy is situated in the direction of Virgo cluster (VC), and is also catalogued as VCC 0241. Its negative radial velocity does not contradict its membership to the cluster (Binggeli et al. 1993). In this case, its distance is of $\approx 20.7 \mathrm{Mpc}$ (distance modulus $=31.6 \mathrm{mag}$, Federspiel et al. 1998). Its maximal diameter on the isophote $\mu_{B}=$ $25^{\mathrm{m}} / \square^{\prime \prime}$ is $111^{\prime \prime}$, according to LEDA, which corresponds to a linear size of about $11 \mathrm{kpc}$. The extent of the $\mathrm{H} \alpha$ emission on our long-slit spectrum is of $70^{\prime \prime}$, which gives the total extent of HiI-emission to be $\approx 6.8 \mathrm{kpc}$.

If $\mathrm{VV} 432$ is a member of $\mathrm{VC}$ it appears to be the most metal-deficient known galaxy of this cluster (see for comparison the metallicity data of the VC BCGs in Izotov \& Guseva 1989). Such low metallicity implies that the galaxy presumably experienced only one or two major SF episodes during its life. Since galaxy interactions in clusters are very important triggers, it is difficult to understand how VV 432 could sustain its very low metallicity in the dense VC environment. The existence of such metal-poor gas-rich galaxy poses serious questions on its evolution history, and thus gives additional arguments to make independent check of its distance, using, in particular, color-magnitude diagrams for resolved stars.

To estimate physical parameters of VV 432 we accept further that this galaxy is in $\mathrm{VC}$, at the distance $20.7 \mathrm{Mpc}$.

Since as we already noticed VV 432 resembles by its morphology an edge-on bent disk, it is natural to check probable companion galaxies, acting as strong enough disturbing bodies.

According to $\mathrm{NED}^{4}$, the nearest galaxies in both the projected distance and the relative velocity are VCC 0200 (MCG +02-31-076) at the angular distance $41.4^{\prime}$ and $V_{\text {hel }}=65 \mathrm{~km} \mathrm{~s}^{-1}$, with $B$-magnitude $=14^{\mathrm{m}} 69$, and

4 The NASA/IPAC Extragalactic Database (NED) is operated by the Jet Propulsion Laboratory, California Institute of Technology, under contract with the National Aeronautics and Space Administration. 
NGC 4216 (UGC 7284) at the angular distance 51.8' and $V_{\text {hel }}=131 \mathrm{~km} \mathrm{~s}^{-1}$, with $B$-magnitude $=10$. 99 . If they are members of $\mathrm{VC}$, their respective projected distances relative to VV 432 are 240 and $312 \mathrm{kpc}$. NGC 4216 according to LEDA is a giant spiral with $M_{B}=-21$ m 6 . If it is indeed on the same radial distance as VV 432, it really can exert strong enough tidal action on VV 432 to trigger gravitational instability and subsequent SF bursts. In particular, tidal generation of shocks in gaseous disk according to Icke (1985) mechanism can be responsible for observed SF bursts (see e.g. Pustilnik et al. 2000, for the estimates of tidal effect in similar situation).

\section{2. $V V 543=N G C 5275$}

This galaxy was considered as a candidate dwarf galaxy $\left(M_{B}=-15^{\mathrm{m}} 5\right)$ due to its catalog radial velocity $V=$ $1395 \mathrm{~km} \mathrm{~s}^{-1}$ (RC3, de Vaucouleurs et al. 1991, cited also in both LEDA and NED, and originally obtained by Arkhipova \& Esipov 1979). However colour indices of this object are rather typical of giant E galaxies than for dIrr systems (Zasov \& Arkhipova 2000). According to our observations, it is evident that there was some misprint in the original work, caused the catalog velocity of this galaxy to be in error by the factor of ten. Its real velocity, measured from our spectrum of emission-line region on the western periphery (hereafter VV 543W) is $14100 \mathrm{~km} \mathrm{~s}^{-1}$.

In fact, the spectrum of the central bright region of this galaxy shows, that we have in this case an optical pair in projection. Indeed the central part shows only the absorption lines, typical of elliptical galaxy (e.g. Pickles 1988), but its radial velocity is $1620 \pm 120 \mathrm{~km} \mathrm{~s}^{-1}$ lower than that for the emission-line galaxy. Its luminosity $M_{B}=-20{ }^{\mathrm{m}} 9$ evidences that this galaxy falls to the class of normal ellipticals. The apparent magnitude of $\mathrm{VV} 543 \mathrm{~W}$ is $\approx 2.5$ fainter $\left(B \approx 17^{\mathrm{m}} \cdot 7\right.$ ) than that of $\mathrm{VV} 543 \mathrm{E}$ (this estimate follows from the comparison of the flux near $\lambda 4400 \AA$ in both galaxies), what leads to $M_{B} \sim-18.7$. Its linear size along the major axis $\sim 13 \mathrm{kpc}$ is quite modest, and, owing to the typical spectrum of HII-region, this western component can be considered as a bright blue compact/HII galaxy. The compact object lying at about $20^{\prime \prime}$ to NE from the absorption-line galaxy is a foreground star.

The extent of $\mathrm{H} \alpha$ emission along the minor axis is traced within $8^{\prime \prime}$. Even after the binning in 4 pixels (which corresponds to the seeing of $2^{\prime \prime}$ ) the velocity curve does not indicate clear gradient. The full range of the radial velocity is about $80 \mathrm{~km} \mathrm{~s}^{-1}$ with the mean value of $14100 \pm 30 \mathrm{~km} \mathrm{~s}^{-1}$.

According to NED, VV 543W has a probable companion galaxy NGP9 F324-0303806 at 5.8' with the radial velocity $13699 \pm 126 \mathrm{~km} \mathrm{~s}^{-1}$ and $B=16{ }^{\mathrm{m}} 68$. Corresponding projection distance $\approx 300 \mathrm{kpc}$ and velocity difference $401 \pm 130 \mathrm{~km} \mathrm{~s}^{-1}$ are in the range typical of wide pairs of binary galaxies (see e.g. the study by Chengalur et al.
1993 and Nordgren et al. 1998). Some weak tidal action from this galaxy can be responsible for the enhanced SF in VV 543W (see e.g. Reshetnikov \& Combes 1997, and Rudnick \& Rix 1998).

\section{3. $V V 747=C G 798$}

This dwarf galaxy consists of two clearly separated regions embedded into the common envelope of low surface brightness. The brighter SW component has full size of about $15^{\prime \prime}$ and the fainter NE can be traced down to about $8^{\prime \prime}(\approx 0.8$ and $0.4 \mathrm{kpc}$ respectively). Both regions show emission-line spectrum (see Fig. 3b). The high excitation spectrum with the observed [OIII] line $\lambda 4363 \AA$ of the SW component allows to determine oxygen abundance using the direct measurement of $T_{\mathrm{e}}$. Our $\mathrm{O}$ abundance is $12+\log (\mathrm{O} / \mathrm{H})=7.85 \pm 0.05$ compared to the abundance 7.97 derived by Izotov \& Thuan (1999). This low metallicity is not untypical for BCGs.

Smooth velocity curve along the slit shows a small but clear slope across the SW component in the region of high $S / N$ ratio of $\mathrm{H} \alpha$ with the total extent of about $15^{\prime \prime}$ and the velocity range from 540 to $630 \mathrm{~km} \mathrm{~s}^{-1}$, consistent with the maximal rotation velocity of about $45 \mathrm{~km} \mathrm{~s}^{-1}$ and mean radial velocity of $585 \mathrm{~km} \mathrm{~s}^{-1}$. The galaxy body is well elongated, so the inclination correction does not seem to be larger than $20 \div 30 \%$. In the NE component the velocity can be measured only in two independent points, showing significant scattering, consistent with their internal uncertainties. The mean velocity of NE component, taking from these two points, is about $690 \pm 42 \mathrm{~km} \mathrm{~s}^{-1}$.

Huchra et al. (1995) presented the values of the observed velocities for two components of this galaxy, designated as SW and NE, but their coordinates given in the paper, are the same for both of them. If we accept that they observed the same components, our results are consistent with theirs $(V(\mathrm{SW})=621 \pm 32$ and $V(\mathrm{NE})=$ $665 \pm 44 \mathrm{~km} \mathrm{~s}^{-1}$ ) within the cited uncertainties.

Since the two separate velocity points for the NE component do not show any clear gradient, which would indicate its independent rotation, and the mean velocity of the NE component matches well the continuation of the velocity curve for the SW component, there is no reason to consider this system as two different galaxies in collision. The current data favour the interpretation of this system as a single galaxy with two super-giant HiI-regions in different excitation stages. In this case the systemic velocity of this single galaxy is about $620 \mathrm{~km} \mathrm{~s}^{-1}$ with the full velocity range of 540 to $710 \mathrm{~km} \mathrm{~s}^{-1}$. Both these values are quite well consistent with the parameters of Hi-profile of VV 747 described and shown in Sect. 3.2. The latter full velocity range corresponds to the maximal rotational velocity of $85 \mathrm{~km} \mathrm{~s}^{-1}$.

The better knowledge of the velocity curve in the region of the NE component is necessary to exclude completely the hypothesis of merger of two dwarf galaxies. 

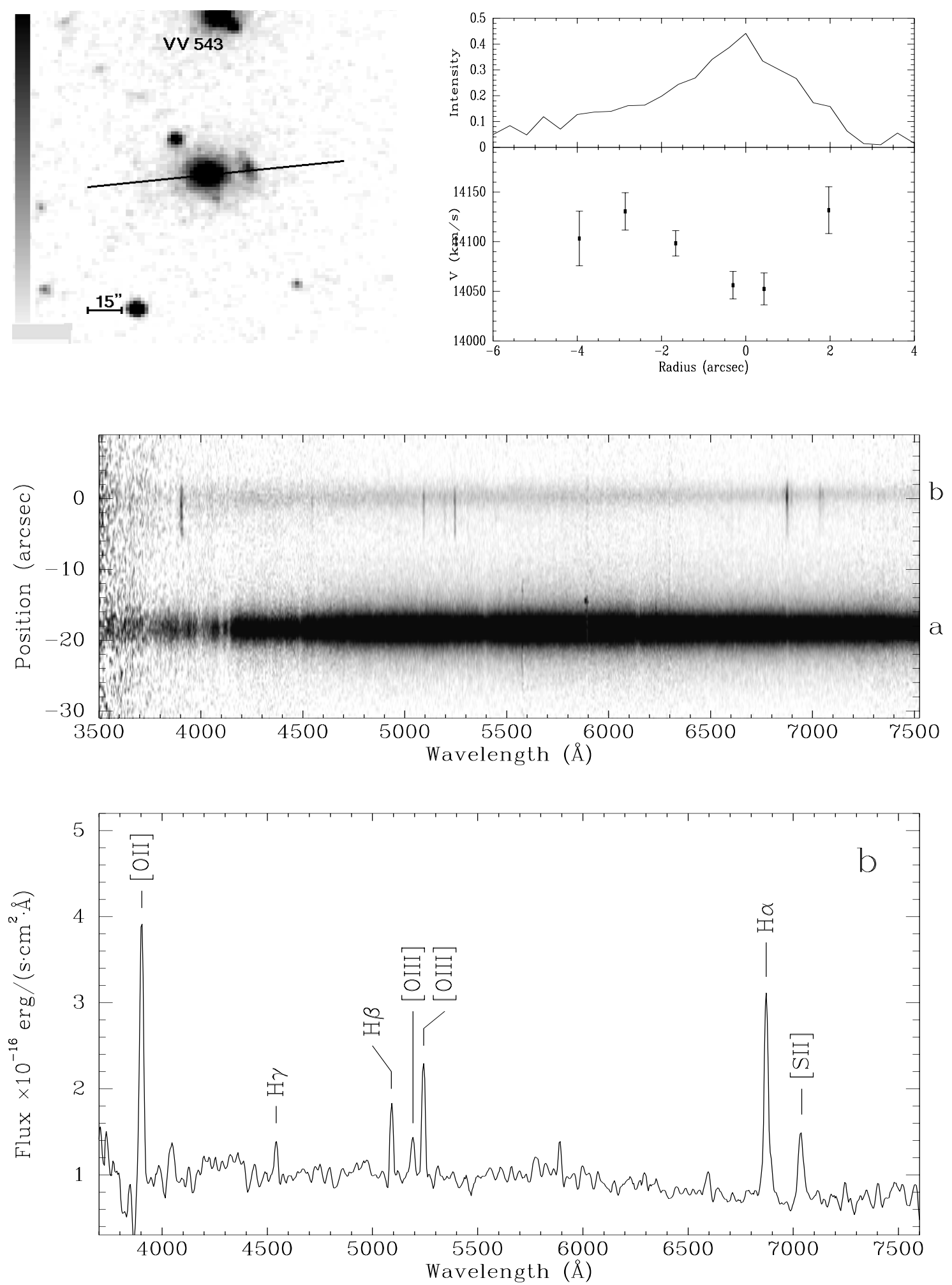

Fig. 2. From top-left to bottom: a) DSS-1 image of galaxy pair VV 543E and VV 543W with the position of long slit superimposed; b) Brightness profile of $\mathrm{H} \alpha$ in relative intensities along the slit and the velocity curve.; c) 2-D spectrum of the pair VV 543; d) 1-D spectrum of the Western component 

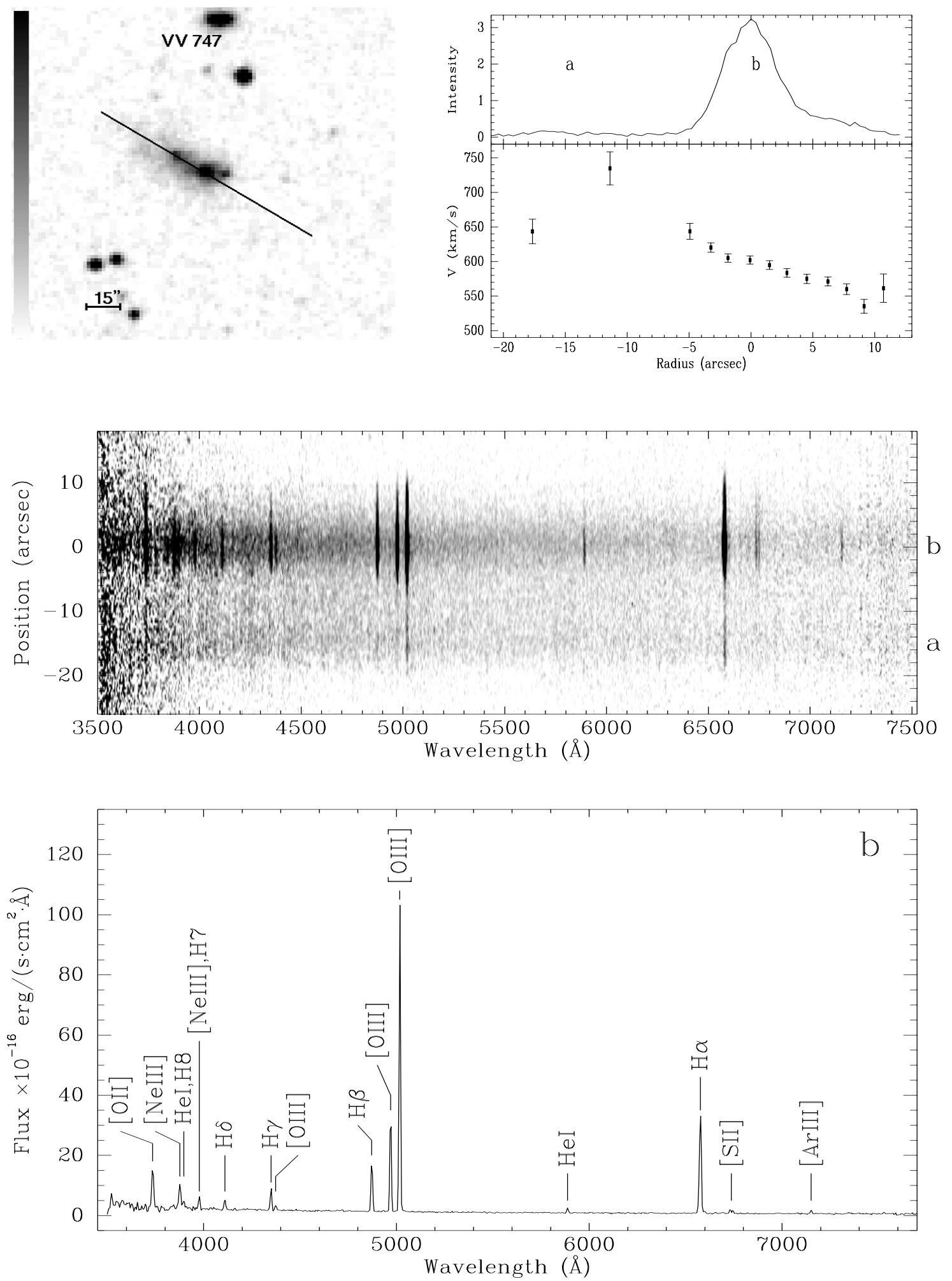

Fig. 3. a) DSS-1 image of galaxy VV 747 with the position of long slit superimposed; b) brightness profile of H $\alpha$ along the slit and the velocity curve of VV 747 ; c) 2-D spectrum of VV 747; d) 1-D spectrum of the brightest SW component 
Table 4. Abundances in VV 432, VV 543W and VV 747

\begin{tabular}{lcccc}
\hline Value & VV 432 (a) $(4.6 \AA /$ pixel $)$ & VV 432 (a) $(1.2 \AA /$ pixel $)$ & VV 747 (a) $(4.6 \AA /$ pixel $)$ & VV $543 W(4.6 \AA /$ pixel $)$ \\
\hline$T_{\mathrm{e}}(\mathrm{O}$ III $)(\mathrm{K})$ & $20680 \pm 1720$ & $18480 \pm 3320$ & $15380 \pm 840$ & $7500 \pm 1500$ \\
$N_{\mathrm{e}}(\mathrm{S}$ II $)\left(\mathrm{cm}^{-3}\right)$ & $<10$ & $<10$ & $110 \pm 239$ & $<10$ \\
$12+\log (\mathrm{O} / \mathrm{H})$ & $7.57 \pm 0.06$ & $7.62 \pm 0.13$ & $7.85 \pm 0.05$ & $8.5 \pm 0.1^{(1)}$ \\
$\log (\mathrm{N} / \mathrm{O})$ & $-1.41 \pm 0.17$ & $-1.50 \pm 0.34$ & $-1.47 \pm 0.16$ & - \\
$\log (\mathrm{Ne} / \mathrm{O})$ & $-0.63 \pm 0.12$ & - & $-0.78 \pm 0.10$ & - \\
\hline
\end{tabular}

(1) - without [OIII]-line $\lambda 4363 \AA$ obtained by the method described in Sect. 2.2.

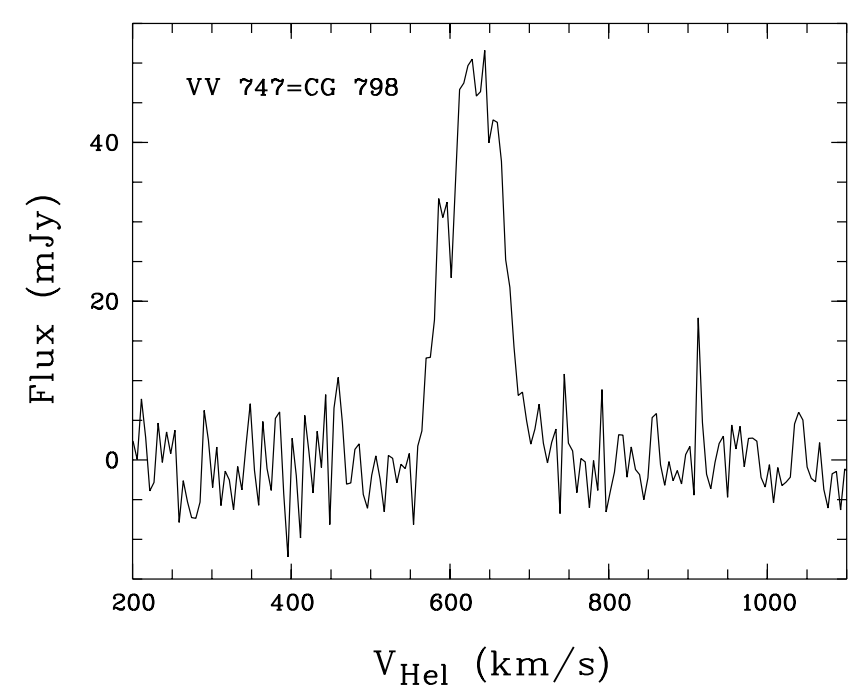

Fig. 4. HI-profile of galaxy VV 747. Velocity resolution is $6.3 \mathrm{~km} \mathrm{~s}^{-1}, W_{0.5}=90 \mathrm{~km} \mathrm{~s}^{-1}, W_{0.2}=117 \mathrm{~km} \mathrm{~s}^{-1}$

At least one indirect argument favours this interpretation. If TF relation holds for this galaxy, its Hi mass and blue luminosity are more consistent with the narrower width $\left(W_{0.2}=70-80 \mathrm{~km} \mathrm{~s}^{-1}\right)$, than with the observed one in the integrated HI-profile. It can indicate that we observe the sum of two "narrow" Hi-profiles displaced at about $40-50 \mathrm{~km} \mathrm{~s}^{-1}$ each from other. Only $21 \mathrm{~cm}$ line mapping of HI velocity field and/or very high quality $\mathrm{H} \alpha$ velocity data can help to make a final interpretation of this system.

The search in the NED resulted in the bright companion galaxy NGC 3432 (= Arp $206=\mathrm{VV}$ 011) at the angular distance $67^{\prime}$ with $B=11^{\mathrm{m}} \cdot 67$ and the radial velocity $616 \mathrm{~km} \mathrm{~s}^{-1}$, very close to that of VV 747 . This galaxy, classified as SB(s)m (LINER Hiı), has $M_{B}=-18$ m 3 . At the projected distance $\approx 190 \mathrm{kpc}$ the tidal action of this galaxy to VV 747 may also be strong enough to trigger $\mathrm{SF}$ in this tiny dwarf.

\section{Discussion and conclusions}

One of the important questions concerning the nature of the "nest" and "chains" of VV-galaxies is their evolution status. Current study as well as several previous publications demonstrate that many of the low-luminosity
VV-galaxies are relatively nearby irregular galaxies with several bright knots of enhanced SF. Their position in two-colour diagram indicates a presence of SF burst in many of them (Zasov \& Arkhipova 2000). They roughly follow so called luminosity - metallicity relation: the least luminous galaxies show in general smaller values of $\mathrm{O} / \mathrm{H}$.

VV 432 has a very low heavy-element abundance. Its $\mathrm{O} / \mathrm{H}$ is among the lowest ten values of the most metaldeficient BCGs out of more than one thousand BCGs/HiIgalaxies known up-to-now. It may be considered as an example of non-evolved galaxy. Analysis of the empirical correlations suggests that dwarf galaxies with $12+\log (\mathrm{O} / \mathrm{H})<7.6$ can currently experience only the first in their history episode of SF (Izotov \& Thuan 1999). Therefore VV 432 with $12+\log (\mathrm{O} / \mathrm{H})=7.58$ is very good candidate for more detailed study. If it is situated in Virgo cluster, it will be the most metal deficient galaxy of this aggregate, being even less chemically evolved than another well-known metal-poor His-galaxy in the direction of Virgo cluster Hi $1225+01$ with $12+\log (\mathrm{O} / \mathrm{H})$ $=7.66$ (Salzer et al. 1991; Chengalur et al. 1995). One of the possible ways to resolve the dilemma of radial distance to VV 432 is a detection of brightest stars and construction of their color-magnitude diagram.

The galaxies we discuss have rather close neighbours. Icke (1985) first has drawn attention to the importance of relatively weak interactions to trigger gravitational instability in gas disks via generation of shocks. Many observational evidences for the important role of weak interactions to trigger SF were obtained since that time, including the detection of low mass HI-companions of nearby HiI-galaxies (Chengalur et al. 1995; Taylor et al. 1993, 1995; Taylor 1997) and optical faint companions of BCGs (Pustilnik et al. 1997). Recent results on late spirals by Reshetnikov \& Combes (1997) and Rudnick \& Rix (1998) also suggest the importance of weak interactions to modulate SF history in these galaxies.

From the observational data discussed above some preliminary conclusions can be drawn:

- Spectrophotometry of VV-galaxies shows that low luminosity representatives of this sample are in general metal-deficient objects, and in this aspect they are similar to dwarf irregular galaxies; 
- The extremely metal-deficient galaxy VV 432 $(12+\log (\mathrm{O} / \mathrm{H})=7.58)$ is probably the least evolved known member of Virgo cluster;

- The system VV 543 radial velocity cited in the RC3 catalog and other databases is wrong. This object consists of two galaxies with discordant redshifts (a unique example among the galaxies of this type!), and probably presents an optical pair. VV 543W is an HiI-galaxy with the radial velocity $1620 \mathrm{~km} \mathrm{~s}^{-1}$ higher than that of absorption-line E-type galaxy VV 543E;

- VV 747 is probably a single dwarf galaxy rich of neutral hydrogen;

- The presence of massive companion galaxies at the distances of few hundred kpc from the studied VV-objects with enhanced SF rate is probably indicative of the important role of weak interactions to trigger SF activity at least in some fraction of low mass VV galaxies.

Acknowledgements. We thank D.Makarov (SAO) for providing us with his MIDAS package to derive velocity curve on longslit spectra. SAO authors appreciate the partial financial support from the RFBR grant No. 96-02-16398 One of the authors (A.Z.) thanks RFBR (grant 98-02-17102) and Federal program "Astronomy" for financial support. We have made use of the NASA/IPAC Extragalactic Database (NED), which is operated by the Jet Propulsion Laboratory, California Institute of Technology, under contract with the National Aeronautics and Space Administration, and of the Lyon-Meudon Extragalactic Database, http://leda.univ-lyon1.fr.

\section{References}

Afanasiev V.L., Karachentsev I.D., Arkhipova V.P., et al., 1980, A\&A 91, 302

Afanasiev V.L., Burenkov A.N., Vlasyuk V.V., Drabek S.V., 1995, SAO RAS internal report, No. 234

Aller L.H., 1984, Physics of Thermal Gaseous Nebulae. Dordrecht: Reidel

Alloin D., Collin-Souffrin S., Joly M., Vigroux L., 1979, A\&A 78,200

Arkhipova V.P., Esipov V.F., 1979, Pis'ma v AZh 5, 265

Arkhipova V.P., Afanasiev V.L., Dostal' V.A., et al., 1981, AZh 58,490

Arkhipova V.P., Noskova R.I., Sil'chenko O.K., Zasov A.V., 1987a, Pis'ma v AZh 13 (7), 575

Arkhipova V.P., Zasov A.V., Noskova R.I., 1987b, AZh 64, 233

Arkhipova V.P., Zasov A.V., Noskova R.I., Sil'chenko O.K., 1987c, AZh 64, 1161

Binggeli B., Popescu C.C., Tamman G.A., 1993, A\&AS 98, 275

Chengalur J.N., Salpeter E.E., Terzian Y., 1993, ApJ 419, 30

Chengalur J.N., Giovanelli R., Haynes M.P., 1995, AJ 109, 2415

de Vaucouleurs G., de Vaucouleurs A., Corwin H.G.Jr., et al., 1991, Third Reference Catalog of Bright Galaxies. Springer-Verlag (RC3)
Federspiel M., Tammann G.A., Sandage A., 1998, ApJ 495, 115

Huchra J.P., Geller J.P., Corwin H.G.Jr., 1995, ApJS 99, 391

Icke V., 1985, A\&A 144, 115

Izotov Y.I., Guseva N.G, 1989, Afz 3, 564

Izotov Y.I., Thuan T.X., Lipovetsky V.A., 1994, ApJ 435, 647 Izotov Y.I., Thuan T.X., Lipovetsky V.A., 1997, ApJS 108, 1

Izotov Y.I., Thuan T.X., 1999, ApJ 511, 639

Kniazev A.Y., Shergin V.S., 1995, SAO RAS internal report No. 249, 1

Kniazev A.Y., Pustilnik S.A., Ugryumov A.V., Kniazeva T.F., 2000, Astron. Lett. 26, 163

Massey P., Strobel K., Barnes J.V., Anderson E., 1988, ApJ 328,315

McGaugh S., 1991, ApJ 380, 140

Nordgren T.E., Chengalur J.N., Salpetert E.E., Terzian Y., 1998, ApJS 115, 43

Olofsson K., 1997, A\&A 321, 29

Pagel B.E.J., Edmunds M.G., Blackwell D.E., Chun M.S., Smith G., 1979, MNRAS 189, 95

Pickles A.J., van der Kruit P.C., 1988, Towards understanding galaxies at large redshift, in: Proc. of the Fifth Workshop of the Advanced School of Astronomy (A89-12501 02-90). Kluwer, Dordrecht, p. 29

Pustilnik S.A., Kniazev A.Y., Ugryumov A.V., 1997, in: IAU XXIII General Assembly, J.D. No. 2 "Dwarf Galaxies", Kyoto (in preparation)

Pustilnik S.A., Brinks E., Thuan T.X., Lipovetsky V.A., Izotov Y.I., 2000, AJ (submitted)

Reshetnikov V., Combes F., 1997, A\&A 324, 80

Rudnick G., Rix H.-W., 1998, BAAS 193, 213

Salzer J.J., Di Serego Alighieri S., Matteucci F., Giovanelli R., Haynes M., 1991, AJ 101, 1258

Schneider S.E., Helou G., Salpeter E.E., Terzian Y., 1986, AJ 92,742

Schneider S.E., Thuan T.X., Magri C., Wadiak J.E., 1991, ApJS 72, 245

Taylor C.L., Brinks E., Skillman E.D., 1993, AJ 105, 128

Taylor C.L., Brinks E., Skillman E.D., 1995, ApJS 99, 427

Taylor C.L., 1997, ApJ 480, 524

Thuan T.X., Lipovetsky V.A., Martin J.-M., Pustilnik S.A., 1999, A\&AS 139, 1

Tully R.B., Fisher J.R., 1977, A\&A 54, 661

Vorontsov-Vel'yaminov B.A., 1959, Atlas and Catalogue of Interacting Galaxies, Part 1. Moscow Univ. Press

Vorontsov-Vel'yaminov B.A., 1977, A\&AS 28, 1

Vorontsov-Vel'yaminov B.A., 1979a, Pis'ma v AZh 5, 499

Vorontsov-Vel'yaminov B.A., 1979b, Pis'ma v AZh 5, 502

Zaritsky D., White S., 1994, ApJ 435, 599

Zaritsky D., Smith R., Frenk C., White S.D.M., 1997, ApJ 478, p. 39 and 53

Zasov A.V., Arkhipova V.P., 2000, in "Small Groups of Galaxies", Proc. IAU Symp. 174 held in Turku, Finland, 1999, Valtonen M. and Flynn C. (eds.). ASP, San-Francisco (in press) 